\title{
Sphingolipids in neuroinflammation: a potential target for diagnosis and therapy
}

\author{
Ju Youn Lee $e^{1,2,3}$, Hee Kyung Jin ${ }^{1,4, *} \mathcal{E} J a e-s u n g ~ B a e^{1,2,3, *}$ \\ ${ }^{1}$ Alzheimer's Disease Research Institute, Kyungpook National University, Daegu 41566, ${ }^{2}$ Department of Physiology, School of Medicine, \\ Kyungpook National University, Daegu 41944, ${ }^{3}$ Department of Biomedical Science, BK21 Plus KNU Biomedical Convergence Program, \\ Kyungpook National University, Daegu 41944, ${ }^{4}$ Department of Laboratory Animal Medicine, College of Veterinary Medicine, Kyungpook \\ National University, Daegu 41566, Korea
}

\begin{abstract}
Sphingolipids are ubiquitous building blocks of eukaryotic cell membranes that function as signaling molecules for regulating a diverse range of cellular processes, including cell proliferation, growth, survival, immune-cell trafficking, vascular and epithelial integrity, and inflammation. Recently, several studies have highlighted the pivotal role of sphingolipids in neuroinflammatory regulation. Sphingolipids have multiple functions, including induction of the expression of various inflammatory mediators and regulation of neuroinflammation by directly effecting the cells of the central nervous system. Accumulating evidence points to sphingolipid engagement in neuroinflammatory disorders, including Alzheimer's and Parkinson's diseases. Abnormal sphingolipid alterations, which involves an increase in ceramide and a decrease in sphingosine kinase, are observed during neuroinflammatory disease. These trends are observed early during disease development, and thus highlight the potential of sphingolipids as a new therapeutic and diagnostic target for neuroinflammatory diseases. [BMB Reports 2020; 53(1): 28-34]
\end{abstract}

\section{INTRODUCTION}

Sphingolipids are mainly present on membrane surface and thereby define the physical and chemical properties of membranes (1). Sphingolipid metabolism involves several intermediate metabolites, including sphingomyelin, ceramide, sphingosine, and sphingosine-1-phosphate (S1P), as well as related enzymes, such as sphingomyelinase (SMase), ceramidase, and sphingosine kinase (SphK), in the biosynthesis

${ }^{*}$ Corresponding authors. Jae-sung Bae, Tel: +82-53-420-4815; Fax: +82-53-424-3349; E-mail: jsbae@knu.ac.kr; Hee Kyung Jin, Tel: +82-53-950-5966; Fax: +82-53-950-5955; E-mail: hkjin@knu.ac.kr

https://doi.org/10.5483/BMBRep.2020.53.1.278

Received 8 October 2019

Keywords: Alzheimer's disease, Ceramide, Neuroinflammation, Parkinson's disease, Sphingosine kinase, Sphingosine-1-phosphate and catabolism of sphingolipids (2). Furthermore, sphingolipids have a rapid turnover, and their levels are controlled by the balance between synthesis and degradation in multiple compartments $(1,2)$. Sphingolipid metabolites are emerging as important signaling molecules that regulate cell growth, survival, immune-cell trafficking, and vascular and epithelial integrity, and are particularly important in inflammation $(1,3)$. Recently, many studies have reported that sphingolipids, together with their corresponding enzymes and receptors, are closely related to the inflammatory process, and have thereby suggested that such sphingolipids are effective drug targets for inflammatory disease treatment $(1,3,4)$.

Inflammation in the central nervous system (CNS) is called neuroinflammation, and it is a major pathogenesis, which affects neurodegenerative disease onset and progression $(5,6)$. Although the ultimate causes of disease development are different, chronic neuroinflammation is a common feature in the progression of several neurodegenerative diseases (5-7). Damage to the CNS parenchyma in several neurodegenerative diseases leads to the activation of microglia and astrocytes and eventually generates a local inflammatory response (8). The activated glial cells clear dead cells and suppress inflammatory molecules, and thereby resolve neuroinflammation via specialized pro-resolving mediators (SPMs) $(9,10)$. However, when resolution of inflammation fails, chronic neuroinflammation, which induces excessive inflammatory cytokines and neuronal death, causes the development of chronic neurodegenerative diseases (5-7).

The brain has the highest sphingolipid content (11), and changes in the brain's lipid levels may initiate pathogenic processes in neuroinflammatory diseases (12). Sphingolipids in the brain may be involved in regulating the key intracellular events of cytokine signaling; producing pro-inflammatory eicosanoids; and modulating cellular mechanisms relevant to apoptosis, astroglial activation, and astrogliosis; increasing T-cell migration; and activating several receptor-mediated pathways (12). Several studies have reported that alterations in brain sphingolipid metabolism are associated with neuroinflammatory diseases including Alzheimer's disease (AD) and Parkinson's diseases (PD) $(13,14)$. Notably, although there is

ISSN: 1976-670X (electronic edition)

Copyright (c) 2020 by the The Korean Society for Biochemistry and Molecular Biology

(ㄷ) This is an open-access article distributed under the terms of the Creative Commons Attribution Non-Commercial License (http://creativecommons.org/licenses/by-nc/4.0) which permits unrestricted non-commercial use, distribution, and reproduction in any medium, provided the original work is properly cited. 
increasing evidence regarding the biological roles of sphingolipid metabolism in neuroinflammation (15), the mechanistic details remain largely unclear. Here, we address the functions and roles of key bioactive sphingolipids in neuroinflammation and discuss the possibility of sphingolipids as therapeutic options for neuroinflammatory disorders.

\section{SPHINGOLIPID METABOLISM IN NEUROINFLAMMATION}

The cells of CNS possess sphingolipids of various patterns, depending on their cell type, location, and pathophysiological conditions $(12,16)$. Sphingolipids are also important in neuroinflammation regulation, and any changes in sphingolipid metabolism occurring in the brain can reportedly cause neuronal cell death (17), glial cell activation (12), and excessive expression of inflammatory molecules (1). Furthermore, several animal models of neuroinflammatory and neurodegenerative diseases, which have a deficit in sphingolipid receptors and enzymes, are employed to demonstrate the functional role of sphingolipid metabolism in neuronal cell death and glial activity (18), cell proliferation (19), and inflammatory mediator production (1). Ceramides were formed in lysosomes by neutral or acid SMase via removal of the head groups of sphingomyelin. Ceramide deacylation by ceramidase is the only pathway to produce sphingosine, and sphingosine can also be reversed to ceramide; this process plays a major role in sphingolipid homeostasis. SphKs, SphK1, and SphK2 phosphorylate sphingosine to generate S1P, which is a key bioactive metabolite in the sphingolipid-to-glycerolipid metabolic pathway (Fig. 1). Among the sphingolipid metabolites, the metabolism of ceramide and S1P especially play major roles in neuroinflammation (20). However, current reports on the beneficial versus detrimental roles of ceramides and S1P in neuroinflammation are controversial; therefore, we have focused our discussion on ceramides and S1P during neuroinflammation.

\section{Ceramide}

Some researchers have reported that ceramides play pro-inflammatory roles in various diseases, such as pulmonary edema, airway inflammation, cystic fibrosis, and inflammatory bowel disease (4), although another study revealed conflicting findings pertaining to anti-inflammatory effects of ceramides in macrophages, neutrophils, and corneal epithelial cells (12). These contradictory reports are also observed during neuroinflammation. Ceramide regulates multiple neuronal cell mechanisms, including cell death, cellular senescence, and cell differentiation in neurons. Increase in ceramide concentration may induce neuronal apoptosis via diverse mechanisms, including direct generation of reactive oxygen species (ROS), mitochondrial dysregulation, and caspase 3 activation $(20,21)$. A study on primary rat astrocytes revealed that ceramide stimulates the expression of pro-inflammatory

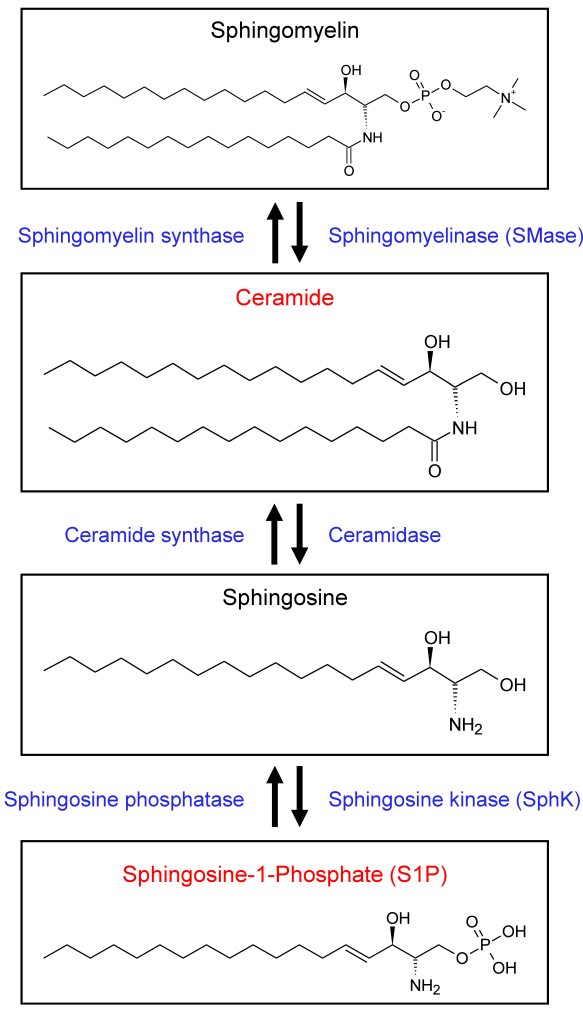

Fig. 1. Sphingolipid metabolism and interconnected bioactive sphingolipid metabolites. The various enzymes that mediate interconversion between various bioactive lipids are localized with the lipids themselves. Ceramide is produced from sphingomyelin by SMase. Ceramide can be hydrolyzed to sphingosine, which is then phosphorylated to sphingosine-1-phosphate (S1P) by sphingosine kinase (SphK)

molecules (TNF- $\alpha$, iNOS, IL-1 $\beta$, and IL-6) and activation of NF- $\kappa B$ (22). Reduction in ceramide via SMase knockdown decreases astrocyte activation in the mouse cortex (23). This finding strongly suggests that increased ceramide concentration in CNS can induce neuronal apoptosis and astrocyte activation as well as play a pro-inflammatory role in neuroinflammatory modulation $(19,24)$. Similar to astrocytes, SMase of microglia produces long-chain ceramides, C16-C24, with the subsequent activation of the pro-inflammatory transcription factor, NF-kB (12). SMase knockdown could also prevent the induction of pro-inflammatory molecules and activation of NF- $\kappa \mathrm{B}$ in microglia, indicating a pro-inflammatory role of ceramide in microglia. Interestingly, short-chain ceramides, which is formed for mimicking the function of naturally occurring long-chain ceramides, exert an antiinflammatory effect on microglia (25). In rodent lipopolysaccharide (LPS)-stimulated microglia, short-chain ceramides inhibit cytokine and chemokines as well as reduce iNOS and cyclooxygenase-2 (COX2) expression and consequently 
decrease ROS level. In the microglia, long-chain ceramides mediate pro-inflammatory effects, whereas short-chain ceramides play an anti-inflammatory role; thus, this findings suggest that the function of ceramides depends on acyl chain length and cell types.

\section{S1P}

S1P plays a prominent role in cellular signaling for maintaining homeostasis, including inflammation, as an intracellular secondary messenger or as an extracellular ligand for the G protein-coupled receptors S1P1-S1P5 (26). CNS cells, including microglia, neurons, and astrocytes, regulate S1P levels via SphK1 and highly express S1PRs, indicating that metabolism and signaling of S1P is associated with neuroinflammation (27). Research regarding the impact of metabolism and signaling of S1P on neuroinflammation was mainly performed in glial cells (27). The reported role of S1P in cell proliferation, migration, and changes in the morphology of astrocytes and microglia suggests a crucial role of S1P in neuroinflammatory conditions $(12,28)$. Cellular activation by LPS stimulates SphK1-S1P signaling, which increases SphK1 expression and continuously generates S1P. This, in turn, causes an elevation of cellular proliferation as well as synthesis of pro-inflammatory cytokines (TNF- $\alpha$, IL- $1 \beta$, and IL-17) and neurotoxic molecules. (1, 29). Although S1P treatment induced a pro-inflammatory response, SphK1 suppression reduced this response; this suggests that the production of these pro-inflammatory mediators directly depended on S1P synthesis in glial cells. It has also been shown that knockdown of SphK or S1P receptor reduces astroglial proliferation and gliosis (30). FTY720, the S1P receptor antagonist, when applied to TNF- $\alpha$-treated human astrocytes, reduced the secretion of monocyte chemotactic protein-1, demonstrating the attenuating effects of proinflammatory response of astrocytes (31). Several studies have suggested that S1P expressed by SphK1 played a role in pro-inflammatory response regulation in glial cells.

\section{SphK}

SphK 1 and 2 are enzymes that generate S1P, which is the key lipid for inflammation regulation, by phosphorylating sphingosine $(1,3,32)$. In a recent study, our group reported the novel roles of SphK in neuroinflammation in the AD brain. Unlike microglial SphK1 described in previous studies (12, 27-29), neuronal SphK1 levels are reduced in neuroinflammatory environments, including $A D$, without any change in the S1P level (33). We confirmed that the reduction in mRNA expression and SphK activity in AD neurons was higher than that in WT neurons, although there was no significant difference in the expression between microglia and astrocytes. However, SphK2 mRNA expression did not differ among the neurons, microglia, and astrocytes derived from WT and AD mice. Sphingosine and S1P levels were also unchanged in these cells. We also confirmed that the comparable lipid levels between AD and WT mice can be attributed to similar SphK2 expression. Reduction in SphK1 causes defective microglial phagocytosis and dysfunctional inflammation resolution owing to the reduced secretion of SPMs, and thereby exacerbates AD pathology. Increased neuronal SphK1 promoted SPM secretion via acetylation of COX2 serine residue 565 (S565), causing an elevation in microglial phagocytic ability. It also restored amyloid- $\beta(A \beta)$ phagocytosis of microglia and cognitive deficits in $\mathrm{AD}$ mice model. These results suggest that SphK1 level was reduced in $A D$ neurons, and the reduction in SphK1, regardless of S1P level, may affect disease progression and/or pathogenesis, including neuroinflammation (Fig. 2) (33). This study also demonstrated a novel effect of SphK1 as acetyltransferase on COX2 expression. SphK1 had acetyl-CoAdependent acetyltransferase activity, showing an increase in COX2 acetylation in the presence of $\left[{ }^{14} \mathrm{C}\right]$ acetyl-CoA. These results revealed the new role of neuronal SphK1, an acetyl-CoA-dependent cytoplasmic acetyltransferase, in COX2 expression. Additionally, this study suggested the specific site of COX2 that is at acetylated by SphK1, namely S565, and the possibility of sphingosine involvement in this reaction. S565 acetylation of COX2 might be induced by sphingosine or a sphingosine intermediate.

When COX2 is acetylated by acetylsalicylic acid (ASA, aspirin) $(34,35)$, the acetylated COX2 produces SPMs from
A

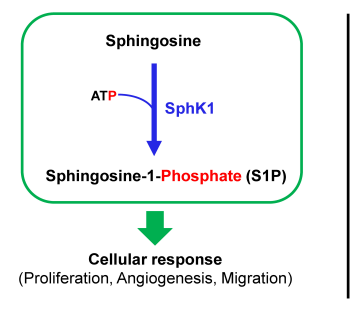

B

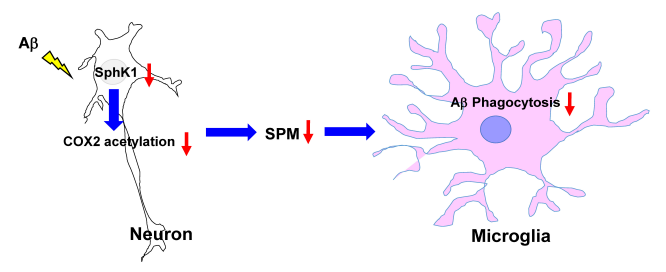

Fig. 2. The role of neuronal SphK1 in neuroinflammation. (A) Left, the role of SphK1 as an ATP-dependent lipid kinase that catalyzes the conversion of sphingosine to S1P, which in turn generates cellular responses including proliferation, angiogenesis, and migration. Right, the novel role of SphK1 as an acetyl-CoA dependent acetyltransferase with activity directed toward COX2, using sphingosine as the substrate. These enzyme activities induce SPM secretion, resulting in the resolution of neuroinflammation. (B) In neuroinflammatory environments, including those with the presence of $A \beta$, neuronal SphK1 decreases, which impairs S565 acetylation of COX2 and reduces SPMs secretion. Decreased SPM secretion in neurons reduced the number of microglia with a phagocytic phenotype. 
arachidonic acid, eicosapentaenoic acid, and docosahexaenoic acid $(36,37)$. SPMs are potent pro-resolving mediators, which reduce immune-cell infiltration and pro-inflammatory factors and increase anti-inflammatory mediators, phagocytosis, and tissue regeneration (36-38). Similar to aspirin-triggered COX2 acetylation, S565 COX2 acetylation by SphK1 produces more SPMs, resulting in the resolution of neuroinflammation and regulation of microglial function (33). These results reveal the novel roles of SphK1 in neuroinflammation, which leads to S565 acetylation of COX2 and secretion of SPMs. Accordingly, we believe that either sphingosine or a sphingosine intermediate participates in this mechanism, which suggests the feasibility of using SphK1-mediated COX2 acetylation as a novel potential therapy for neuroinflammatory diseases, including $A D$, in the future.

Overall, these findings suggest that sphingolipids are implicated in neuroinflammation in different pathophysiological conditions $(1,3)$. Ceramide is increased in neuroinflammatory environments and is associated with neuronal apoptosis and glial activation $(12,24)$. During neuroinflammation, the acyl chain length of ceramides is different in microglia, in that the long-chain and short-chain ceramides mediate pro-inflammatory and anti-inflammatory effects, respectively $(12,39)$. S1P is also elevated by SphK1 in glial cells during neuroinflammation, which consequently stimulates pro-inflammatory responses (26). During neuroinflammation, neuronal SphK1 level reduces, which, in turn, decreases S565 COX2 acetylation and SPM secretion, and thereby causes the failure of neuroinflammatory resolution (33). All these findings suggest that sphingolipids play a pivotal role in neuroinflammation and

\section{Neuroinflammatory Environments}

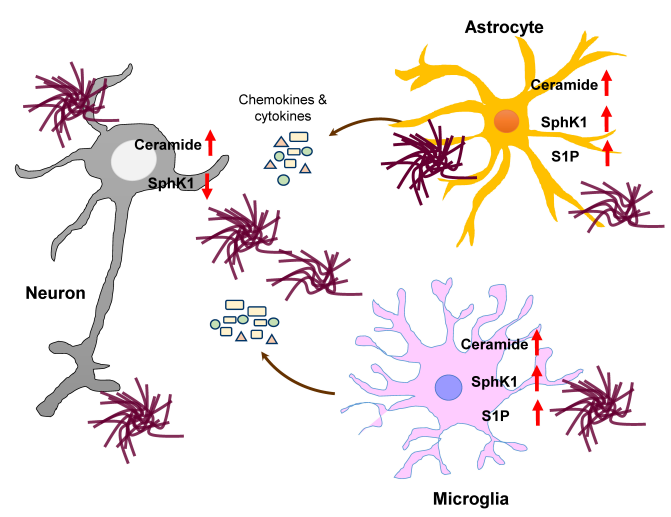

Fig. 3. Changes in bioactive sphingolipid levels observed during neuroinflammation. Numerous observations in neuroinflammatory environments demonstrate changes in sphingolipid levels. Ceramide is increased in the neurons and glial cells of neuroinflammatory environments. S1P expressed by SphK1 is elevated in the neuroinflammatory environments of glial cells. Neuronal SphK is reduced, regardless of S1P alternations. should therefore be considered in the etiological studies of neuroinflammation and neurodegenerative diseases as well as their progression (Fig. 3).

\section{POTENTIAL THERAPEUTIC INTERVENTION IN SPHINGOLIPID-INDUCED NEUROINFLAMMATION}

Several experiments have revealed the importance of sphingolipids in neuroinflammation. The discovery of sphingolipid mechanism stimulated considerable clinical interest on sphingolipid metabolites and their role in cell signaling during neuroinflammatory diseases (1). This suggests that bioactive sphingolipids are associated with neuroinflammatory diseases and may be potential therapeutic targets. Furthermore, alteration of sphingolipid metabolism during early disease stage might prove to be potential biomarkers of neurodegenerative disorders.

\section{Alzheimer's disease}

$\mathrm{AD}$ is the most common cause of dementia and is characterized by cognitive decline, caused by the accumulation of extra- and intra-cellular $A \beta$ plaques and hyperphosphorylated tau (40, 41). The studies conducted on postmortem brain tissues have revealed excessive chronic inflammation in the plaque area. A key phenomenon underlying AD-related neuroinflammation is the proliferation and accumulation of microglial cells around plaques, which results in the expression of pro-inflammatory cytokines and chemokines as well as neuronal cell death (42-44).

$A \beta$ increases ceramide levels by activating SMase, and thereby contributes to apoptosis. For AD pathogenesis, sphingomyelin hydrolysis induced by $A \beta$ is the main source of ceramides, followed by an increase in SMase level during AD progression (45). Moreover, an imbalance in ceramide metabolism, which potentially decides the fate of brain cells, reportedly occurs during early stage of $\mathrm{AD}$. Thus, anti-ceramide immunity might suggest a novel potential diagnosis and therapy for neuroinflammatory diseases, including AD (20). Moreover, SphKs and S1P levels can be downregulated by A $\beta$ $(20,33)$. The S1P levels were reduced with an increasing Braak stage in tissue samples obtained from the CA1 region of the hippocampus or from the gray and white matter areas of the inferior temporal gyrus (46). AD brain shows an increase in S1P lyase and S1P-metabolizing phosphatases (47). In our study, we showed that SphK1 but not S1P expression was reduced in $A D$ neurons (33). Many researchers have demonstrated a reduction in SphK level during $A D$, indicating the importance of SphK in AD progression and/or pathogenesis.

Salter et al. and Ulland et al. reported microglial dysfunction, including elevated pro-inflammatory markers and defective phagocytic function in $A D(48,49)$. The capacity of microglial phagocytosis in $\mathrm{AD}$ can be improved, which may provide a novel approach to AD therapy in the future $(50,51)$. In $\mathrm{AD}$ neurons, we reported the loss of SphK1 activity as well 
as a reduction in S565 COX2 acetylation and SPM secretion, which led to a decrease in microglial phagocytosis and caused the failure of inflammatory resolution (33). Overexpression of SphK1 in AD mice increased phagocytosis and anti-inflammatory markers, and decrease in pro-inflammatory marker levels occurred owing to the restoration of microglial function via COX2 acetylation-derived SPMs. During AD, these results suggest that neuronal SphK1 regulates microglial function via COX2 acetylation and SPM secretion (33). Furthermore, on conducting further studies, we discovered an intermediate between SphK1 and COX2 in SphK1-mediated COX2 acetylation, which ultimately led to SPM secretion. This intermediate resolved neuroinflammation and positively regulated microglia, which in turn caused an improvement in AD pathology. This suggests that the intermediate of SphK1-mediated COX2 acetylation affects microglial function and phenotype; thus, it may be a promising agent for $A D$ treatment.

COX2 is the main enzyme involved in an inflammatory environment, which induces the synthesis of pro-inflammatory prostaglandins and cytokines (52). Therefore, COX2 inhibition was considered to be a therapeutic strategy for inflammatory diseases, including AD (53). Among the various COX2 inhibitors, only aspirin can generate SPMs via COX2 acetylation as well as block pro-inflammatory pathway via COX2 inhibition (54). However, the use of aspirin on AD patients did not improve pathology $(55,56)$, and these results can be attributed to multiple functions of aspirin, including inhibition of COX1 and COX2 as well as COX2 acetylation (57). Recently, our study demonstrated that COX2 acetylation decreased in AD neurons, and it is modulated by neuronal SphK1. Although neuronal SphK1 regulates COX2 acetylation and SPM secretion, it did not affect the expression of COX2 mRNA and protein (33). These results suggest that SphK1-mediated COX2 acetylation is a novel therapeutic strategy for neuroinflammatory diseases such as AD.

When $A \beta$ and tau are deposited at a high concentration in the brain, positron emission tomography is used for detecting $A \beta$ and tau aggregates in order to diagnose $A D$ (58). However, it is difficult to diagnose the disease before the proteins aggregate. Alteration in sphingolipids begins to occur at very early stages of $\mathrm{AD}$ pathogenesis, perhaps before amyloid plaques are formed; this suggests that sphingolipids may be a promising therapeutic target and useful in the diagnosis of $A D$ at preclinical stages. Oligomerized $A \beta$ peptides in $\mathrm{AD}$ brain promote ceramide formation during early AD stages (20). Moreover, SphK1 deceases before $A \beta$ aggregation occurs. In thioflavin $S$ staining, $A \beta$ plaques begin to appear in AD mice at the age of 6 six months, which consequently leads to a reduction in neuronal SphK1 level and activation of glial cell in AD mice (33). These results indicated that reduction in neuronal SphK1 occurred before $A \beta$ aggregation, which led to activation of glial cells in APP/PS1 mice; hence sphingolipid metabolites, including ceramide and
SphK1, can be considered for diagnosing neuroinflammation at early AD stages. Several studies regarding sphingolipids and $A D$ have suggested that sphingolipids, including ceramide and SphK, can be useful and accessible AD biomarkers as well as potential therapeutic targets.

\section{Parkinson's disease}

$\mathrm{PD}$ is a complex neurodegenerative disorder that mainly affects the motor system, causing bradykinesia, rigidity, tremors, postural instability, chronic fatigue, anxiety, and apathy. PD is characterized by the progressive loss of dopaminergic neurons in the substantia nigra and striatum. The main histopathological hallmark of PD is the aggregation of diverse proteins such as $\alpha$-synuclein in CNS cells, which results in the formation of Lewy bodies and Lewy neurites. Although the loss of dopaminergic neurons was the principal cause of PD, glial activation that occurred during nigrostriatal dopaminergic system damage has been proved to be a major factor for PD progression (59-62).

Recent researches have presented the correlation between sphingolipids and PD, although further studies are warranted. Disturbances in sphingolipid metabolism, including altered ceramides and SphK1 levels, are reported in postmortem PD brain tissues. The ceramide levels were significantly higher, but the expression and activity of SphK1 was reduced in PD (20). Interestingly, PD is similar to AD in terms of disturbances in sphingolipid metabolism. Based on the studies conducted on $A D$, ceramide inhibition and induction of SphK1-mediated COX2 acetylation can be proposed as a possible means of PD therapy.

\section{CONCLUSION}

In this review, we obtained the biophysical and biochemical properties of sphingolipids in neuroinflammation, and they can be used in new therapeutic and diagnostic approaches for diverse neuroinflammatory diseases. Sphingolipids have gained popularity because several researches have highlighted their implications in neuroinflammation. This dramatic increase in the understanding of sphingolipids and neuroinflammation has led to the development of several novel drugs. In neuroinflammatory diseases, such as AD and PD, the levels of ceramides and SphK are altered, which impacted disease pathogenesis. We propose that ceramide inhibition and induction of SphK1-mediated COX2 acetylation are potential targets for the diagnosis and therapy of neuroinflammatory disease. Future researches can guide drug development for regulating specific sphingolipid-mediated functions that are important in the pathogenesis of human neuroinflammatory diseases.

\section{ACKNOWLEDGEMENTS}

This research was supported by the Basic Science Research 
Program (2017R1A4A1015652) and the Original Technology Research Program for Brain Science (2018M3C7A1056513) of the NRF funded by the Korean government, MSIT. This research was also supported by a grant of the Korea Health Technology R\&D Project through the KHIDI, funded by the Ministry of Health \& Welfare, Republic of Korea (HI16C2131).

\section{CONFLICTS OF INTEREST}

The authors have no conflicting interests.

\section{REFERENCES}

1. Maceyka M and Spiegel S (2014) Sphingolipid metabolites in inflammatory disease. Nature 510, 58-67

2. Merrill AH Jr (2011) Sphingolipid and glycosphingolipid metabolic pathways in the era of sphingolipidomics. Chem Rev 111, 6387-6422

3. Hannun YA and Obeid LM (2018) Sphingolipids and their metabolism in physiology and disease. Nat Rev Mol Cell Biol 19, 175-191

4. Nixon GF (2009) Sphingolipids in inflammation: pathological implications and potential therapeutic targets. Br J Pharmacol 158, 982-993

5. Chitnis T and Weiner HL (2017) CNS inflammation and neurodegeneration. J Clin Invest 127, 3577-3587

6. Brambilla R (2019) Neuroinflammation, the thread connecting neurological disease : Cluster: "Neuroinflammatory mechanisms in neurodegenerative disorders". Acta Neuropathol 137, 689-691

7. Schwartz M and Baruch K (2014) The resolution of neuroinflammation in neurodegeneration: leukocyte recruitment via the choroid plexus. EMBO J 33, 7-22

8. Norris GT and Kipnis J (2019) Immune cells and CNS physiology: Microglia and beyond. J Exp Med 216, 60-70

9. Arcuri C, Mecca C, Bianchi R, Giambanco I and Donato R (2017) The Pathophysiological Role of Microglia in Dynamic Surveillance, Phagocytosis and Structural Remodeling of the Developing CNS. Front Mol Neurosci 10,191

10. Zhu M, Wang X, Hjorth E et al (2016) Pro-Resolving Lipid Mediators Improve Neuronal Survival and Increase A 342 Phagocytosis. Mol Neurobiol 53, 2733-2749

11. Kosicek M and Hecimovic S (2013) Phospholipids and Alzheimer's disease: alterations, mechanisms and potential biomarkers. Int J Mol Sci 14, 1310-1322

12. Assi E, Cazzato D, De Palma C, Perrotta C, Clementi E and Cervia D (2013) Sphingolipids and brain resident macrophages in neuroinflammation: an emerging aspect of nervous system pathology. Clin Dev Immunol 2013, 309302

13. Liu Q and Zhang J (2014) Lipid metabolism in Alzheimer's disease. Neurosci Bull 30, 331-345

14. Yin F, Sancheti H, Patil I and Cadenas E (2016) Energy metabolism and inflammation in brain aging and Alzheimer's disease. Free Radic Biol Med 100, 108-122

15. Davies L, Fassbender K and Walter S (2013) Sphingolipids in neuroinflammation. Handb Exp Pharmacol 216,
421-430

16. Mesa-Herrera F, Taoro-González L, Valdés-Baizabal C, Diaz M and Marín R (2019) Lipid and Lipid Raft Alteration in Aging and Neurodegenerative Diseases: A Window for the Development of New Biomarkers. Int J Mol Sci 20, E3810

17. Di Pardo A and Maglione V (2018) Sphingolipid Metabolism: A New Therapeutic Opportunity for Brain Degenerative Disorders. Front Neurosci 12, 249

18. Szepesi Z, Manouchehrian O, Bachiller S and Deierborg T (2018) Bidirectional Microglia-Neuron Communication in Health and Disease. Front Cell Neurosci 12, 323

19. Young MM, Kester M and Wang HG (2013) Sphingolipids: regulators of crosstalk between apoptosis and autophagy. J Lipid Res 54, 5-19

20. Czubowicz $K$, Jeśko $H$, Wencel $P$, Lukiw $W J$ and Strosznajder RP (2019) The Role of Ceramide and Sphingosine-1-Phosphate in Alzheimer's Disease and Other Neurodegenerative Disorders. Mol Neurobiol 56, 5436-5455

21. Jęśko H, Stępień A, Lukiw WJ and Strosznajder RP (2019) The Cross-Talk Between Sphingolipids and Insulin-Like Growth Factor Signaling: Significance for Aging and Neurodegeneration. Mol Neurobiol 56, 3501-3521

22. Pahan K, Sheikh FG, Khan M, Namboodiri AM and Singh I (1998) Sphingomyelinase and ceramide stimulate the expression of inducible nitric-oxide synthase in rat primary astrocytes. J Biol Chem 273, 2591-2600

23. Liu L, Martin R and Chan C (2013) Palmitate-activated astrocytes via serine palmitoyltransferase increase BACE1 in primary neurons by sphingomyelinases. Neurobiol Aging 34, 540-550

24. de Wit NM, den Hoedt S, Martinez-Martinez P, Rozemuller AJ, Mulder MT and de Vries HE (2019) Astrocytic ceramide as possible indicator of neuroinflammation. J Neuroinflammation 16, 48

25. Jung JS, Shin KO, Lee YM et al (2013) Anti-inflammatory mechanism of exogenous C2 ceramide in lipopolysaccharide-stimulated microglia. Biochim Biophys Acta 1831, 1016-1026

26. Fischer I, Alliod C, Martinier N, Newcombe J, Brana C and Pouly S (2011) Sphingosine kinase 1 and sphingosine 1-phosphate receptor 3 are functionally upregulated on astrocytes under pro-inflammatory conditions. PLoS One 6, e23905

27. Grassi S, Mauri L, Prioni S et al (2019) Sphingosine 1-Phosphate Receptors and Metabolic Enzymes as Druggable Targets for Brain Diseases. Front Pharmacol 10,807

28. Karunakaran I and van Echten-Deckert G (2017) Sphingosine 1-phosphate - A double edged sword in the brain. Biochim Biophys Acta Biomembr 1859, 1573-1582

29. Spiegel $S$ and Milstien $S$ (2011) The outs and the ins of sphingosine-1-phosphate in immunity. Nat Rev Immunol $11,403-415$

30. Wu YP, Mizugishi K, Bektas M, Sandhoff R and Proia RL (2008) Sphingosine kinase 1/S1P receptor signaling axis controls glial proliferation in mice with Sandhoff disease. Hum Mol Genet 17, 2257-2264

31. Rothhammer V and Quintana FJ (2015) Control of 
autoimmune CNS inflammation by astrocytes. Semin Immunopathol 37, 625-638

32. Chalfant CE and Spiegel S (2005) Sphingosine 1-phosphate and ceramide 1-phosphate: expanding roles in cell signaling. J Cell Sci 118, 4605-4612

33. Lee JY, Han SH, Park MH et al (2018) Neuronal SphK1 acetylates COX2 and contributes to pathogenesis in a model of Alzheimer's Disease. Nat Commun 9, 1479

34. Kalgutkar AS, Crews BC, Rowlinson SW, Garner C, Seibert K and Marnett LJ (1998) Aspirin-like molecules that covalently inactivate cyclooxygenase-2. Science 280, $1268-1270$

35. Müller N (2019) COX-2 Inhibitors, Aspirin, and Other Potential Anti-Inflammatory Treatments for Psychiatric Disorders. Front Psychiatry 10, 375

36. Serhan CN (2014) Pro-resolving lipid mediators are leads for resolution physiology. Nature 510, 92-101

37. Buckley CD, Gilroy DW and Serhan CN (2014) Proresolving lipid mediators and mechanisms in the resolution of acute inflammation. Immunity 40, 315-327

38. Fullerton JN, O'Brien AJ and Gilroy DW (2014) Lipid mediators in immune dysfunction after severe inflammation. Trends Immunol 35, 12-21

39. Mencarelli C and Martinez-Martinez P (2013) Ceramide function in the brain: when a slight tilt is enough. Cell Mol Life Sci 70, 181-203

40. Graham WV, Bonito-Oliva A and Sakmar TP (2017) Update on Alzheimer's Disease Therapy and Prevention Strategies. Annu Rev Med 68, 413-430

41. Cerquera-Jaramillo MA, Nava-Mesa MO, González-Reyes RE, Tellez-Conti C and de-la-Torre A (2018) Visual Features in Alzheimer's Disease: From Basic Mechanisms to Clinical Overview. Neural Plast 2018, 2941783

42. Heppner FL, Ransohoff RM and Becher B (2015) Immune attack: the role of inflammation in Alzheimer disease. Nat Rev Neurosci 16, 358-372

43. Visan I (2017) Alzheimer's disease microglia. Nat Immunol 18, 876

44. Katsumoto A, Takeuchi H, Takahashi $\mathrm{K}$ and Tanaka F (2018) Microglia in Alzheimer's Disease: Risk Factors and Inflammation. Front Neurol 9, 978

45. Jazvinšćak Jembrek M, Hof PR and Šimić G (2015) Ceramides in Alzheimer's Disease: Key Mediators of Neuronal Apoptosis Induced by Oxidative Stress and $A \beta$ Accumulation. Oxid Med Cell Longev 2015, 346783

46. Couttas TA, Kain N, Daniels B et al (2014) Loss of the neuroprotective factor Sphingosine 1-phosphate early in Alzheimer's disease pathogenesis. Acta Neuropathol Commun 2, 9

47. Ceccom J, Loukh N, Lauwers-Cances V et al (2014)
Reduced sphingosine kinase- 1 and enhanced sphingosine 1-phosphate lyase expression demonstrate deregulated sphingosine 1-phosphate signaling in Alzheimer's disease. Acta Neuropathol Commun 2, 12

48. Salter MW and Stevens B (2017) Microglia emerge as central players in brain disease. Nat Med 23, 1018-1027

49. Ulland TK and Colonna M (2018) TREM2- a key player in microglial biology and Alzheimer disease. Nat Rev Neurol $14,667-675$

50. Fakhoury M (2018) Microglia and Astrocytes in Alzheimer's Disease: Implications for Therapy. Curr Neuropharmacol 16, 508-518

51. Kim SH, Noh MY, Kim HJ et al (2019) A Therapeutic Strategy for Alzheimer's Disease Focused on Immuneinflammatory Modulation. Dement Neurocogn Disord 18, 33-46

52. Alhouayek M and Muccioli GG (2014) COX-2-derived endocannabinoid metabolites as novel inflammatory mediators. Trends Pharmacol Sci 35, 284-292

53. Bertolini A, Ottani A and Sandrini M (2002) Selective COX-2 inhibitors and dual acting anti-inflammatory drugs: critical remarks. Curr Med Chem 9, 1033-1043

54. Chiang N and Serhan CN (2004) Aspirin triggers formation of anti-inflammatory mediators: New mechanism for an old drug. Discov Med 4, 470-475

55. Kotilinek LA, Westerman MA, Wang Q et al (2008) Cyclooxygenase-2 inhibition improves amyloid-betamediated suppression of memory and synaptic plasticity. Brain 131, 651-664

56. Mitchell JA and Warner TD (2006) COX isoforms in the cardiovascular system: understanding the activities of non-steroidal anti-inflammatory drugs. Nat Rev Drug Discov 5, 75-86

57. Xu XM, Sansores-Garcia L, Chen XM, Matijevic-Aleksic N, Du M and Wu KK (1999) Suppression of inducible cyclooxygenase 2 gene transcription by aspirin and sodium salicylate. Proc Natl Acad Sci U S A 96, 5292-5297

58. Cuello AC (2017) Early and Late CNS Inflammation in Alzheimer's Disease: Two Extremes of a Continuum? Trends Pharmacol Sci 38, 956-966

59. Poewe W, Seppi K, Tanner CM et al (2017) Parkinson disease. Nat Rev Dis Primers 3, 17013

60. Engelender $S$ and Isacson O (2017) The Threshold Theory for Parkinson's Disease. Trends Neurosci 40, 4-14

61. Charvin D, Medori R, Hauser RA and Rascol O (2018) Therapeutic strategies for Parkinson disease: beyond dopaminergic drugs. Nat Rev Drug Discov 17, 844

62. McGregor MM and Nelson AB (2019) Circuit Mechanisms of Parkinson's Disease. Neuron 101, 1042-1056 